\title{
Design and Development of Mobile, Tablet-based ECG Hardware and Software for Clinical Use
}

\author{
${ }^{1}$ Chen, H., ${ }^{1}$ Yang, Y., ${ }^{1}$ Wallace, JR., ${ }^{2}$ Jiang, N. and ${ }^{1}$ Mainville, M. \\ ${ }^{1}$ School of Public Health and Health Systems \\ ${ }^{2}$ System Design Engineering \\ University of Waterloo \\ Waterloo, Ontrio, Canada \\ \{helen.chen,y24yang,jrwallace,ning.jiang\}@waterloo.ca,mel.mainville@gmail.com
}

\begin{abstract}
The exising technologies used to capture Electrocardiograms (ECGs) pose a number of challenges, including bulky hardware, slow processing times, and inadequate built-in support for ensuring the quality of collected data. We worked with a major healthcare organization to develop mobile, tablet-based ECG hardware and software. In this work, we describe requirements gathering, design, development activities for our mobile ECG data collection solutions, followed by an initial user testing. The results of this research show the potential of mobile device-based ECGs in the clinical domain, and point to types of automation that the healthcare industry may resist and a need for care in automating the collection of ECGs.
\end{abstract}

\section{CCS CONCEPTS}

- Human-centered computing $\rightarrow$ User centered design;

\section{KEYWORDS}

miniaturized ECG, mobile data collection, ECG signal processing, user evaluation, data quality

\section{ACM Reference Format:}

${ }^{1}$ Chen, H., ${ }^{1}$ Yang, Y., ${ }^{1}$ Wallace, JR., ${ }^{2}$ Jiang, N. and ${ }^{1}$ Mainville, M.. 2019. Design and Development of Mobile, Tablet-based ECG Hardware and Software for Clinical Use. In Proceedings of ACM Conference on PervasiveHealth (PervasiveHealth '19). ACM, New York, NY, USA, Article 4, 4 pages. https://doi.org/10.1145/nnnnnnn.nnnnnnn

\section{INTRODUCTION}

The traditional, centralized healthcare services model is moving towards an emphasis on preventative healthcare, and towards care in the community and at home. The mobilization of these services presents an opportunity to revisit how health care is delivered, and to understand how medical devices and workflow can be optimized to support care outside of medical facilities. One of the most common medical tests is an electrocardiogram (ECG or EKG). A clinical grade ECG is obtained by placing 10 electrodes on the skin surrounding the heart to record its electrical impulses, and is used by cardiologists to diagnose conditions such as arrhythmia, myocardial

Permission to make digital or hard copies of part or all of this work for personal or classroom use is granted without fee provided that copies are not made or distributed for profit or commercial advantage and that copies bear this notice and the full citation on the first page. Copyrights for third-party components of this work must be honored.

For all other uses, contact the owner/author(s).

PervasiveHealth '19, May 2019, Trento, Italy

(c) 2019 Copyright held by the owner/author(s).

ACM ISBN 123-4567-24-567/08/06.

https://doi.org/10.1145/nnnnnnn.nnnnnnn

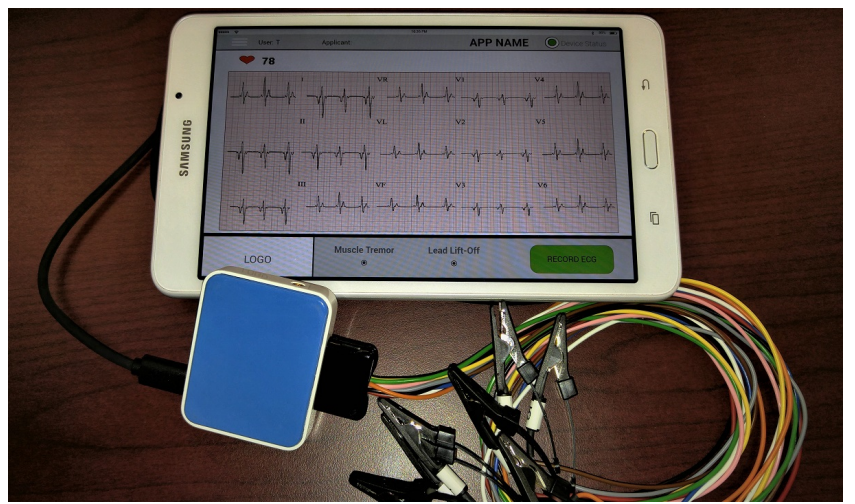

Figure 1: In this work, we developed miniaturized mobile ECG device and a tablet-based ECG data collection app that can simplify existing ECG collection workflows, reduce human error and latency between data collection and diagnosis, and improve security.

infraction (heart attack) or abnormal sinus rhythm. Current mobile ECG technologies are often clumsy to transport, awkward to use, and fraught with data acquisition challenges. Many clinical grade 'mobile' ECG acquisition tools are simply scaled-down versions of devices originally designed for hospital use, making the equipment ill-suited for non-hospital environments.

To address these issues, we developed a miniaturized ECG hardware and mobile app that takes advantage of commodity mobile devices, such as smartphones and tablets, to facilitate data collection in the field (Figure 1). By working with AlphaCare, a large, national health organization to design the solution, we were able to include a range of healthcare professionals in the design process, from technicians in the field collecting samples to cardiologists in an office. We present our design and development process, and conclude with a reflection on the prototype and on opportunities for the use of mobile devices to support healthcare tasks.

\section{PROBLEM OVERVIEW}

According to the World Health Organization [2], the global ECG equipment market is valued at over USD $\$ 5.0$ billion, with a demographic shift towards an older population and increases in cardiac disease expected to increase that market to USD $\$ 6.3$ billion by 2022. Our partner, AlphaCare, a major healthcare service provider, supports more than 10 million Canadians through their clinics and 
data collection facilities. AlphaCare's contracted mobile health professionals (HPs) is expected to perform over 300,000 ECG tests annually under two major divisions: long term care and insurance.

Currently, the majority of ECG testing for both divisions is performed using stand-alone mobile ECG equipment that produces a paper tracing. AlphaCare management revealed that on average, the time between ECG acquisition and diagnosis is around three to ten business days. Inefficiencies with the paper process can result in significant delays, high cost, and decreased satisfaction with services. There is a pressing need for more efficient means of capturing the data. However, several limitations of existing technologies, such as data quality issues, high capital costs and lack of scalability, lack of flixibility and integration, and complex user interfaces have prevented AlphaCare from expanding and improving their mobile services.

To address these limitations, AlphaCare sought out a new solution for mobile ECG capture. Our initial consultations with AlphaCare employees pointed to mobile devices, and in particular smartphones and tablets, being an appropriate platform through which the above limitations may be addressed.

\section{RELATED WORK}

Over the past few years, many new mobile ECG devices have made ECGs more accessible to individuals at home, and have enabled them to collect data that in the past was impossible to collect. The manufacturers of these devices have also developed their own cloudbased data pipeline, and thus it is difficult for large healthcare providers like AlphaCare to integrate their use into existing workflow and data management processes.

In the academic literature, the use of mobile devices has been explored to address this gap. For example, Wang et al. [15] explored the use of wearable sensors in combination with a patient's smartphone to upload long-term ECG data to the cloud. Researchers have explored mobile support for a variety of activities, such as stress management at home [13, 14], physical training [12], and home monitoring for seniors $[1,9]$. However none of these projects have investigated the collection of clinical-grade ECG data. In a clinical context, Hsieh and Hsu [4] developed a smartphone app that uploads data to the cloud after exporting it from an existing ECG device. [7] developed prototypes that enabled wireless transmission of ECG data for emergency contexts, but using a participatory design methodology identify a number of technological limitations may prohibit the adoption of these prototypes in practice.

There have also been significant advancements in signal processing and machine learning, which help to improve data quality. For example, automatic detection of QRS complexes (e.g., [5, 8]) - the characteristic up-down pattern in an ECG - and arrhythmia [6] or other changes in cardiac rhythm [10], and statistical analysis and removal of noise [3] have been refined to the point where they can be tightly integrated with clinical decision support systems (CDSS) [11] to provide real-time feedback to HPs. Many in the healthcare industry, including AlphaCare, desire to leverage these advances to improve patient care through more rapid diagnosis, a reduction in data collection errors, and a more streamlined work flow.

\section{REQUIREMENTS GATHERING}

To understand AlphaCare's business requirements, we first conducted a series of in-person interviews with their Chief Information Officer, Director of Cardiology Operations, front-line Health Professionals (HPs), training staff, and technical support staff. We discussed their organizational structure, current ECG collection process and business needs, and industry and government regulations and policies that affect ECG services. Based on these interviews, user stories and data flow diagrams were developed to assist in specifying the following user and business requirements: 12-lead ECG, compact, Android-compatible, secure, decision support, ease of use, and integration support.

Based on these requirements, we developed a prototype of the mECG hardware and data collection software. We worked closely with AlphaCare's process specialists including managers from LongTerm Care and Homecare, Cardiology Services, Insurance System Analysts, and System Development and Integration team members. Our design was iteratively refined through AlphaCare's feedback, and the prototype was then used to define granular technical requirements of the mECG system used for initial development of the application.

\section{OUR SYSTEM}

Our prototype mECG system is composed of 1) a miniaturized 12Lead ECG microcontroller that connects to a user's smartphone or tablet via micro-USB, 2) an Android application that supports the collection, inspection, and transmission of ECG data.

\subsection{Prototype Hardware}

We developed a custom microcontroller with built-in functionality for signal collection, amplification, and processing (Figure 2). To satisfy AlphaCare's requirements for portability, the total size of our hardware is $50 \mathrm{~mm} \times 40 \mathrm{~mm} \times 8 \mathrm{~mm}$, smaller than a standard deck of playing cards. Furthermore, to increase signal quality, data transfer is through USB connection rather than Bluetooth. In-house testing has been performed on the device to validate its accuracy and precision, and demonstrates results within $0.1 \%$ of existing standards.

The hardware also includes built-in support for two real-time features for improved data quality at the time of collection: detection of electrode signal quality, and motion artifact rejection. Electrode signal quality is helpful in providing a warning to HPs when an electrode may begin to detach or otherwise fail. These signals are passed to our software package so that the HP can be alerted to then double-check the acquired signals. Research and testing during development of the microcontroller showed that independent component analysis (ICA) was particularly effective [3], since artifacts in the multi-sensor ECG setting can be modelled as statistically independent and with non-Gaussian distributions.

\subsection{Prototype Software}

Our prototype app supports data collection, verification, and transmission to AlphaCare's LIS. Throughout the design process, we considered how adopting a mobile device-based interface can help to improve upon existing workflows to address AlphaCare's primary concerns. 

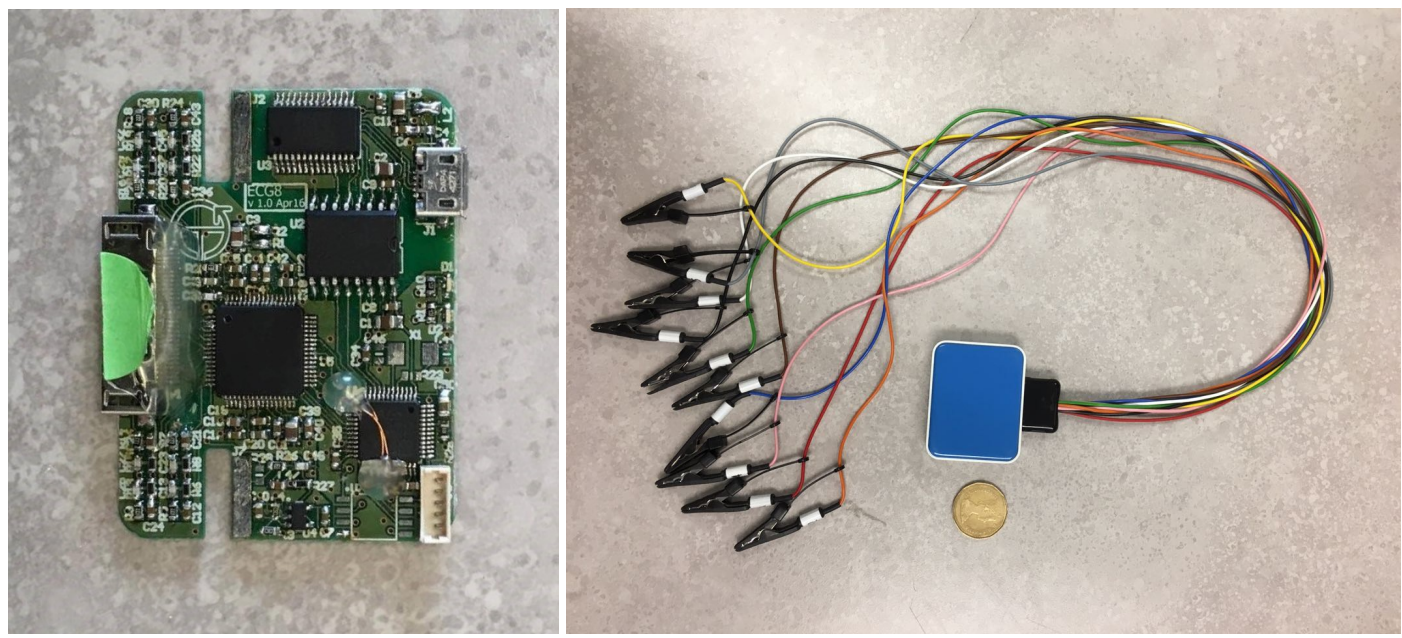

Figure 2: Our prototype hardware includes a (left) custom microcontroller that is encased in a (right) 3D-printed case with support for 10-leads and USB connectivity to a mobile device.

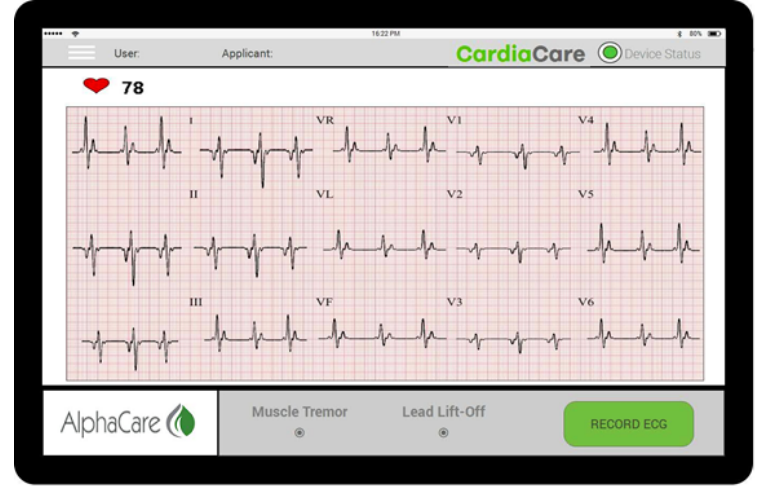

Figure 3: One prototype screen mirrors the existing paperbased metaphor used by HPs at AlphaCare today.

5.2.1 Implementation. Our mECG software is written in Java for Android devices. Our app was optimized to work on the Samsung Galaxy Tab A 7" tablet with 1.3 GHz Quad Core Processor running the latest version of Android Lollipop (5.1). Once tested, algorithms were re-written as native Java libraries and integrated with the Android app.

5.2.2 Data Collection. To support data collection, our app encompasses AlphaCare's existing workflow, including user authentication, requisition form processing for both long-term care and insurance duties, and ECG recording. We augment the paper ECG readout with a simple button to start/stop recording, summary heart rate calculations, and notifications for muscle tremors and lead lift-off, discussed next as supports for data verification and decision support.

5.2.3 Verification and Decision Support. As ECG signals are captured and displayed to the user within the app's interface, real-time notification of quality issues such as muscle tremors and lead-lift off are displayed (Figure 3, Panel 4). Given that users may not be well versed in ECG interpretation, our app also provides features to help an HP determine the quality of the tracing. These features include providing notification of artifacts that can affect ECG quality such as muscle tremors, noise contamination from nearby equipment, and if leads were misplaced or reversed.

5.2.4 Data Transmission and Processing. Our app securely stores all collected ECG data on-device until the HP is able to transfer the data to AlphaCare's LIS. ECG data is transmitted to the client's back-end systems and converted to an ECG report in the desired format (e.g., DICOM, PDF, TIFF). The DICOM format allows data to be processed by the Picture Archive and Communication Systems (PACS) within the LIS, providing economical storage, retrieval, management, and presentation of medical images and reports from multiple modalities such as radiology, ultrasound, magnetic resonance (MR), and mammograms.

\section{INITIAL USER TESTING}

We conducted a lab study in which four experts from AlphaCare used our prototype device to perform a typical ECG recording session. The initial user testing followed a user-centric design and was largely qualitative in nature, a focus group discussion facilitated by the researchers. It started with a brief introduction and a demonstration of the $\mathrm{mECG}$ prototype. As a group, participants were asked to use the provided ECG equipment and app prototype to complete two recordings on a volunteer test subject. Participants were asked structured open-ended questions and responses were audio recorded for research purposes.

The overall impression of our $\mathrm{mECG}$ prototype system and app was positive. In comparison to other ECG equipment they have used, they find that our prototype was very easy to use. In particular, equipped with lots of error detection algorithms and built-in system to eliminate baseline artifact, it automatically removes many errors and distractions by eliminating choices. The participants liked the following design of the app: i) the ability to control it from a remote 
location; ii) easy to fix/troubleshoot from a farther location; and iii) very easy to use settings and realize issues that may cause problems oneself compared to asking a patient who may not know what is wrong with the device.

In the meantime, the following aspects were identified for improvement. Leads, clips and wires got tangled easily. The clips should be larger and thicker, mid-clips or the button type of leads were preferred over the clips. The participants would rather prefer labels on the leads, instead of a standard color coating. The length of the wire should be at least 5 feet and more sturdy with shield. The participants preferred to have the option of sitting or lying down to before, and they would like to be able to take multiple ECGs and choose from the best one to upload. The printing font should be larger on the clips.

\section{DISCUSSION AND FUTURE WORK}

Through the requirements gathering and prototyping process described in this work, we have shown the promise of mobile devices to replace existing, paper-based workflows, to reduce latency between ECG collection and interpretation, and to support HPs in reducing errors in collected ECG data. In particular, AlphaCare was enthusiastic about the potential of commodity, touch-based mobile devices to give non-technical users access to the advanced signal processing tools provided by our hardware, such as $50 \mathrm{~Hz}$ filtering, and the detection of missing leads, wandering baselines, and muscle tremors.

Interestingly, AlphaCare was also resistant to many, more advanced features being introduced to our software. For example, the capability to support feature recognition within the ECG was considered, but ultimately rejected. Similarly, features to automatically correct artifacts in ECG data, such as those introduced by electrical interference, were rejected in favour of making HPs aware of the issues, which they would then manually correct.

While we have addressed the most common concerns identified by AlphaCare in this initial design exercise, we foresee opportunities to develop more advanced features and further improve data collection. For example, we are currently exploring built-in decision-support for diagnosis, where signal processing and machine learning techniques can help detect characteristics such as arrhythmia in an ECG, and alert an HP to take appropriate action. As part of this process, we will be performing a security audit of the device and cloud-based services, ongoing usability and user acceptance testing, and field trials with the device.

\section{CONCLUSION}

We worked with a large, national health care organizations to explore how mobile devices can be used to improve their ECG collection workflow. We developed custom hardware and software to address a number of our partner's requirements, significantly reducing the latency between data collection and interpretation, and providing built-in support to detect and reduce errors in collected data. This work highlights the role that mobile devices can play in the modernization in the healthcare sector, particularly by enterprise companies such as AlphaCare. The ubiquity of smartphones and tablets provides a unique opportunity for these devices to play a role in supporting work by a large workforce, and our work demonstrates significant advantages for industry, such as reduced errors and a reduction in the overall time and effort required to collect and process health data.

\section{ACKNOWLEDGMENTS}

This project is funded by Natural Sciences and Engineering Research Council of Canada CRDPJ 503545-16, Ontario Centre of Excellence 27101 and Dynacare-Gamma Laboratory Partnership

\section{REFERENCES}

[1] S. Dagtas, Y. Natchetoi, and H. Wu. 2007. An Integrated Wireless Sensing and Mobile Processing Architecture for Assisted Living and Healthcare Applications. In Proceedings of the 1st ACM SIGMOBILE International Workshop on Systems and Networking Support for Healthcare and Assisted Living Environments (HealthNet '07). ACM, New York, NY, USA, 70-72. DOI : http://dx.doi.org/10.1145/1248054. 1248074

[2] Grandview-Research. 2016. ECG Equipment Market Size To Reach 6.3 Billion By 2022. (2016). http://http://www.grandviewresearch.com/press-release/ global-ecg-equipment-market

[3] Taigang He, Gari Clifford, and Lionel Tarassenko. 2006. Application of independent component analysis in removing artefacts from the electrocardiogram. Neural Computing \& Applications 15, 2 (2006), 105-116.

[4] Jui-chien Hsieh and Meng-Wei Hsu. 2012. A cloud computing based 12-lead ECG telemedicine service. BMC medical informatics and decision making 12, 1 (2012), 77.

[5] S. Karpagachelvi, M. Arthanari, and M. Sivakumar. 2010. ECG Feature Extraction Techniques - A Survey Approach. CoRR abs/1005.0957 (2010). http://arxiv.org/ abs/1005.0957

[6] Baljit Kaur and Sanjay Singla. 2016. ECG Analysis with Signal Classification Using Decision Tree Induction (DTI). In Proceedings of the International Conference on Advances in Information Communication Technology \& Computing (AICTC '16). ACM, New York, NY, USA, Article 95, 6 pages. DOI : http://dx.doi.org/10.1145/ 2979779.2979874

[7] Morten Kyng, Esben Toftdahl Nielsen, and Margit Kristensen. 2006. Challenges in Designing Interactive Systems for Emergency Response. In Proceedings of the 6th Conference on Designing Interactive Systems (DIS '06). ACM, New York, NY, USA, 301-310. DOI : http://dx.doi.org/10.1145/1142405.1142450

[8] Cuiwei Li, Chongxun Zheng, and Changfeng Tai. 1995. Detection of ECG characteristic points using wavelet transforms. IEEE Transactions on biomedical Engineering 42, 1 (1995), 21-28.

[9] Andreas Lorenz, Dorit Mielke, Reinhard Oppermann, and Lars Zahl. 2007. Personalized Mobile Health Monitoring for Elderly. In Proceedings of the 9th International Conference on Human Computer Interaction with Mobile Devices and Services (MobileHCI '07). ACM, New York, NY, USA, 297-304. DOI: http: //dx.doi.org/10.1145/1377999.1378022

[10] Fabien Massé, Martien Van Bussel, Aline Serteyn, Johan Arends, and Julien Penders. 2013. Miniaturized Wireless ECG Monitor for Real-time Detection of Epileptic Seizures. ACM Trans. Embed. Comput. Syst. 12, 4, Article 102 (July 2013), 21 pages. DOI : http://dx.doi.org/10.1145/2485984.2485990

[11] Mark A Musen, Blackford Middleton, and Robert A Greenes. 2014. Clinical decision-support systems. In Biomedical informatics. Springer, 643-674.

[12] Nuria Oliver and Fernando Flores-Mangas. 2006. MPTrain: A Mobile, Music and Physiology-based Personal Trainer. In Proceedings of the 8th Conference on Humancomputer Interaction with Mobile Devices and Services (MobileHCI '06). ACM, New York, NY, USA, 21-28. DOI : http://dx.doi.org/10.1145/1152215.1152221

[13] Pablo Paredes and Matthew Chan. 2011. CalmMeNow: Exploratory Research and Design of Stress Mitigating Mobile Interventions. In CHI '11 Extended Abstracts on Human Factors in Computing Systems (CHI EA '11). ACM, New York, NY, USA, 1699-1704. DOI : http://dx.doi.org/10.1145/1979742.1979831

[14] Pedro Sanches, Kristina Höök, Elsa Vaara, Claus Weymann, Markus Bylund, Pedro Ferreira, Nathalie Peira, and Marie Sjölinder. 2010. Mind the Body!: Designing a Mobile Stress Management Application Encouraging Personal Reflection. In Proceedings of the 8th ACM Conference on Designing Interactive Systems (DIS '10). ACM, New York, NY, USA, 47-56. DOI : http://dx.doi.org/10.1145/1858171. 1858182

[15] Xiaoliang Wang, Qiong Gui, Bingwei Liu, Zhanpeng Jin, and Yu Chen. 2014. Enabling smart personalized healthcare: a hybrid mobile-cloud approach for ECG telemonitoring. IEEE journal of biomedical and health informatics 18, 3 (2014), 739-745. 